\section{BRAZIULIAN JOURNAL \\ OF MEDICAL AND BIOLOGICAL RLSF.ARCH}

www.bjournal.com.br
ISSN 0100-879X

Volume 42 (12) 1119-1247 December 2009

BIOMEDICAL SCIENCES

AND

CLINICAL INVESTIGATION

Braz J Med Biol Res, December 2009, Volume 42(12) 1143-1149

\title{
Effects of strain and age on ear wound healing and regeneration in mice
}

R.A. Costa, V. Ruiz-de-Souza, G.M. Azevedo Jr., N.M. Vaz and C.R. Carvalho

The Brazilian Journal of Medical and Biological Research is partially financed by
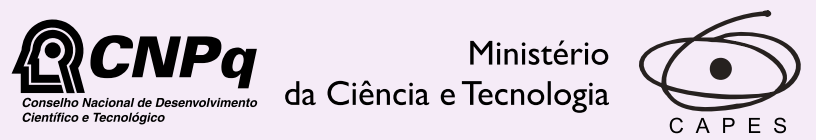

Ministério da Educação

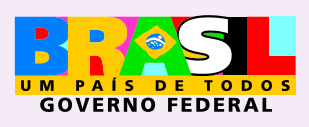

DFAPESP

Institutional Sponsors 


\title{
Effects of strain and age on ear wound healing and regeneration in mice
}

\author{
R.A. Costa1 , V. Ruiz-de-Souza1, G.M. Azevedo Jr. ${ }^{1}$, \\ N.M. Vaz ${ }^{2}$ and C.R. Carvalho ${ }^{1}$
}

1Departamento de Morfologia, ${ }^{2}$ Departamento de Bioquímica-Imunologia, Instituto de Ciências Biológicas, Universidade Federal de Minas Gerais,

Belo Horizonte, MG, Brasil

\begin{abstract}
Round holes in the ears of MRL mice tend to close with characteristics of regeneration believed to be absent in other mouse strains (e.g., C57BL/6). We evaluated the kinetics and the histopathology of ear wound closure in young (8 weeks old) C57BL/6 and BALB/c mice. We also used middle-aged (40 weeks old) C57BL/6 mice to evaluate the influence of aging on this process. A circular through-and-through hole was made in the ear, photographs were taken at different times after injury and wound area was measured with digital analysis software. The percentages of closed area measured on day 100 were: $23.57 \pm 8.66 \%$ for young BALB/c mice, $56.47 \pm 7.39 \%$ for young C57BL/6 mice, and $75.31 \pm 23.65 \%$ for middle-aged C57BL/6 mice. Mice were sacrificed on days $1,3,5,25,44$, and 100 for histological evaluation with hematoxylin and eosin, Gomori's trichrome, periodic acid-Schiff, or picrosirius red staining. In young mice of both strains, healing included re-epithelialization, chondrogenesis, myogenesis, and collagen deposition. Young C57BL/6 and BALB/c mice differed in the organization of collagen fibers visualized using picrosirius-polarization. Sebaceous glands and hair follicles regenerated and chondrogenesis was greater in young C57BL/6 mice. In middle-aged C57BL/6 mice all aspects of regeneration were depressed. The characteristics of regeneration were present during ear wound healing in both young BALB/c and young C57BL/6 mice although they differed in intensity and pattern. Greater ear wound closure in middle-aged C57BL/6 mice was not correlated with regeneration.
\end{abstract}

Key words: Wound healing; Regeneration; Scar; Aging; C57BL/6 mice; BALB/c mice

\section{Introduction}

Organ and tissue regeneration after accidental injury or surgical interventions in humans is a dreamed possibility in the medical and scientific communities. Although vertebrates such as Urodela amphibians can regenerate an amputated limb, adult mammals, in general, have a limited regenerative capacity (1). For most injuries, repair reduces a once functional tissue to a patch of non-functional cells (mainly fibroblasts) and a disorganized extracellular matrix (mainly collagen) that is commonly referred to as a scar. On the other hand, embryos and early fetuses can reconstitute skin after injury without scar tissue formation.

Interestingly, the liver is one of the few organs of adult mammals that can regenerate up to $70 \%$ of itself without scar formation (2). Also, adult rabbits are capable of completely closing a 1-cm diameter through-and-through ear hole, regenerating skin, hair, blood vessels, nerves, muscles, and cartilage (3). A similar event of regeneration was later serendipitously discovered in MRL mice that completely closed, within 30 days, 2-mm through-and-through ear holes generally used for lifelong identification of the animals (4). Many studies on ear hole closure have used C57BL/6 mice from different sources as a control non-regenerating strain and this strain has been reported to have a limited or even null capacity of regenerating ear structures (4-6). Some reports have associated differences in the process of wound healing with differences in immunological activities and have suggested that the occurrence of more robust inflammatory reactions with the participation of lymphocytes impairs regeneration (1,2,7-10). Although many studies have been devoted to this subject, no clear explanation of differences in wound healing or regeneration is available and efforts should be made to obtain it.

Aging is another aspect associated with impaired wound healing. Immunological activities are known to be reduced

Correspondence: C.R. Carvalho, Departamento de Morfologia, ICB-UFMG, Av. Antônio Carlos, 6627, $31270-901$ Belo Horizonte, MG, Brasil. Fax: +55-31-3409-2771. E-mail: crochac@icb.ufmg.br

Research supported by FAPEMIG (CBB 727/06 and CBB 1732/05). R.A. Costa is the recipient of a doctoral scholarship from FAPEMIG.

Received March 16, 2009. Accepted August 21, 2009. Available online November 23, 2009. Published December 4, 2009. 
with aging (11) and age-related alterations in healing might be associated with these altered immunological activities. On the other hand, differences in the skin wound healing process associated with aging may also be related to alterations in skin morphology and function (12).

In the present study, we compared the ear healing process in young ( 8 weeks old) C57BL/6 and BALB/c mice. We made a circular hole in the center of the right ear of unmanipulated naive mice and evaluated histologically the kinetics of ear wound closure at different time intervals after wounding. Unexpectedly, we found some characteristics of regeneration in naive young ( 8 weeks old) C57BL/6 and $\mathrm{BALB} / \mathrm{c}$ mice but with quantitative and qualitative differences between strains. We further studied the healing process in middle-aged (40 weeks old) C57BL/6 mice and noticed that, although displaying a greater percentage of ear hole closure, they showed no sign of regeneration.

\section{Material and Methods}

\begin{abstract}
Animals
Young (8 weeks old) male BALB/c mice and young and middle-aged (40 weeks old) C57BL/6 mice bred and maintained in the animal facilities of Instituto de Ciências Biológicas, Universidade Federal de Minas Gerais (UFMG), Brazil, under non-specific pathogen-free conditions, were used. These stocks were originally derived from breeding pairs obtained from the Jackson Laboratory more than 30 years ago. The animals were fed, housed, and treated according to the guidelines of the Ethics Committee of Animal Experimentation of the UFMG. Each experimental group contained 5-6 mice.
\end{abstract}

\section{Wound}

A through-and-through hole was punched in the center of the cartilaginous part of the right ear using a 1.91-mm diameter metal ear punch (Thomas Scientific, USA; cat No. 1111 W11 ear punch, 2-mm x 2" L). Mice were lightly anesthetized, their ears were flattened against a contrasting background and photographs of wounds in the presence of a scaled ruler were taken with a digital camera (Sony DSCF717, Japan) immediately after wounding. Photographs were repeated at different times from day 2 to day 100 . For quantification of the wound area, digital pictures were analyzed with the Image Tool 3.0 software (Uthscsa, USA, http://ddsdx.uthscsa.edu/dig/itdesc.htm). Measurements of wound area obtained immediately after punching resulted in an average of $2.86 \mathrm{~mm}^{2}$ with no difference between strains. This value was used to calculate the percent variation of wound area throughout the experiments $(\mathrm{N}=5-6$ mice $)$.

\section{Histology}

Animals were killed by cervical dislocation at different intervals after wounding, the ears were removed with scissors by cutting at the base of the pinna, fixed in buffered formalin, $\mathrm{pH} 7.0$, embedded in paraffin, cut into 5- $\mu \mathrm{m}$ thick sections and stained with hematoxylin and eosin, Gomori's trichrome, or periodic acid-Schiff. Digital images of the slides were obtained using an Olympus $Q$ color 3 camera coupled to a BX50 Olympus microscope (Olympus T2, Japan) connected to a computer with the aid of the Q-Capture Pro software ( $Q$ Imaging, Canada).

\section{Picrosirius red staining}

After hydration, the slides containing the $5-\mu \mathrm{m}$ sections were stained for $1 \mathrm{~h}$ in $0.1 \%$ Sirius Red F3BA(BDH Laboratory Supplies, UK) in saturated picric acid. They were placed in $0.1 \mathrm{~N} \mathrm{HCl}$ for 4 min and counterstained with Harris's hematoxylin for $50 \mathrm{~s}$ at room temperature. After washing in water, the sections were dehydrated through an ethanol and xylene series and mounted with synthetic resin. Picrosirius red-stained sections were evaluated by ordinary polychromatic and polarized light microscopy (13). For the analysis of collagen deposition, Picrosirius red-stained sections were observed and digitalized with a BX51 Olympus microscope equipped with a circular polarizer filter (Olympus T2). All slides were photographed on the same day to avoid any variability associated with the light source.

\section{Statistical analysis}

Statistical analyses were performed using the GraphPad Prism 4 software (GraphPad, USA) and the statistical significance of differences between groups for wound closure area was determined by the unpaired Student $t$-test or by one-way ANOVA followed by the Student-Newman-Keuls test. Values of $P \leq 0.05$ were considered to be significant. Data are reported as mean \pm SD percent wound closure area or wound area in $\mathrm{mm}^{2}$.

\section{Results}

\section{Young BALB/c and young C57BL/6 mice: kinetics of ear wound closure}

Immediately after wounding the punched area corresponded to $2.86 \mathrm{~mm}^{2}$ (1.91 $\mathrm{mm}$ in diameter) and there was no difference between the mouse strains. Measurements of wound closure areas were chosen instead of diameters because closure of the wound hole is not regular. The kinetics of wound area variation in relation to the original hole area was similar in young C57BL/6 and BALB/c mice except at day 2, when a decrease in wound area was observed in young C57BL/6 mice $\left(2.18 \pm 0.44 \mathrm{~mm}^{2}\right)$ and an increase was observed in young BALB/c mice (3.12 \pm 0.52 $\left.\mathrm{mm}^{2}, \mathrm{P}<0.05\right)$. At day 5 , the average area of the holes was wider than immediately after wounding $\left(3.30 \pm 0.44 \mathrm{~mm}^{2}\right.$ in C57BL/6 mice and $3.98 \pm 0.38 \mathrm{~mm}^{2}$ in BALB/c mice, $P$ $<0.05)$, being progressively reduced thereafter up to day $15\left(1.37 \pm 0.36 \mathrm{~mm}^{2}\right.$ in C57BL/6 mice and $2.24 \pm 0.36 \mathrm{~mm}^{2}$ in $B A L B / c$ mice, $P<0.05)$ and then stabilizing. There were significant differences in wound closure between the two 
strains from day 2 up to the end of the experiment on day $100\left(1.32 \pm 0.21 \mathrm{~mm}^{2}\right.$ in C57BL/6 mice and $2.20 \pm 0.22 \mathrm{~mm}^{2}$ in BALB/c mice, $\mathrm{P}<0.05)$, when the percentages of closed area measured were $56.47 \pm 7.39 \%$ in young $C 57 \mathrm{BL} / 6$ mice and $23.57 \pm 8.66 \%$ in young BALB/c mice.

\section{Young BALB/c and young C57BL/6 mice: histological differences}

The histology of wound regions at days 1, 3, 5, and 25 after the lesion is shown in Figure 1. At day 1 (Figure $1 A, B)$, we did not observe a difference between strains; necrosis was present and some edema and neutrophil and macrophage infiltration were found near the edges of the hole. At day 3 (Figure 1C,D, insets), fibrin clots had shed from the wounds of most BALB/c mice while they were still attached to the wounds of C57BL/6 mice. At day 3, both strains presented increased edema, some muscle degeneration and extracellular matrix deposition. The process of wound re-epithelialization was almost complete in both strains, but the epidermis was thicker in C57BL/6 mice than in BALB/c mice (Figure 1C,D). By day 5 (Figure 1E,F insets), all clots had shed from the wounds of both strains
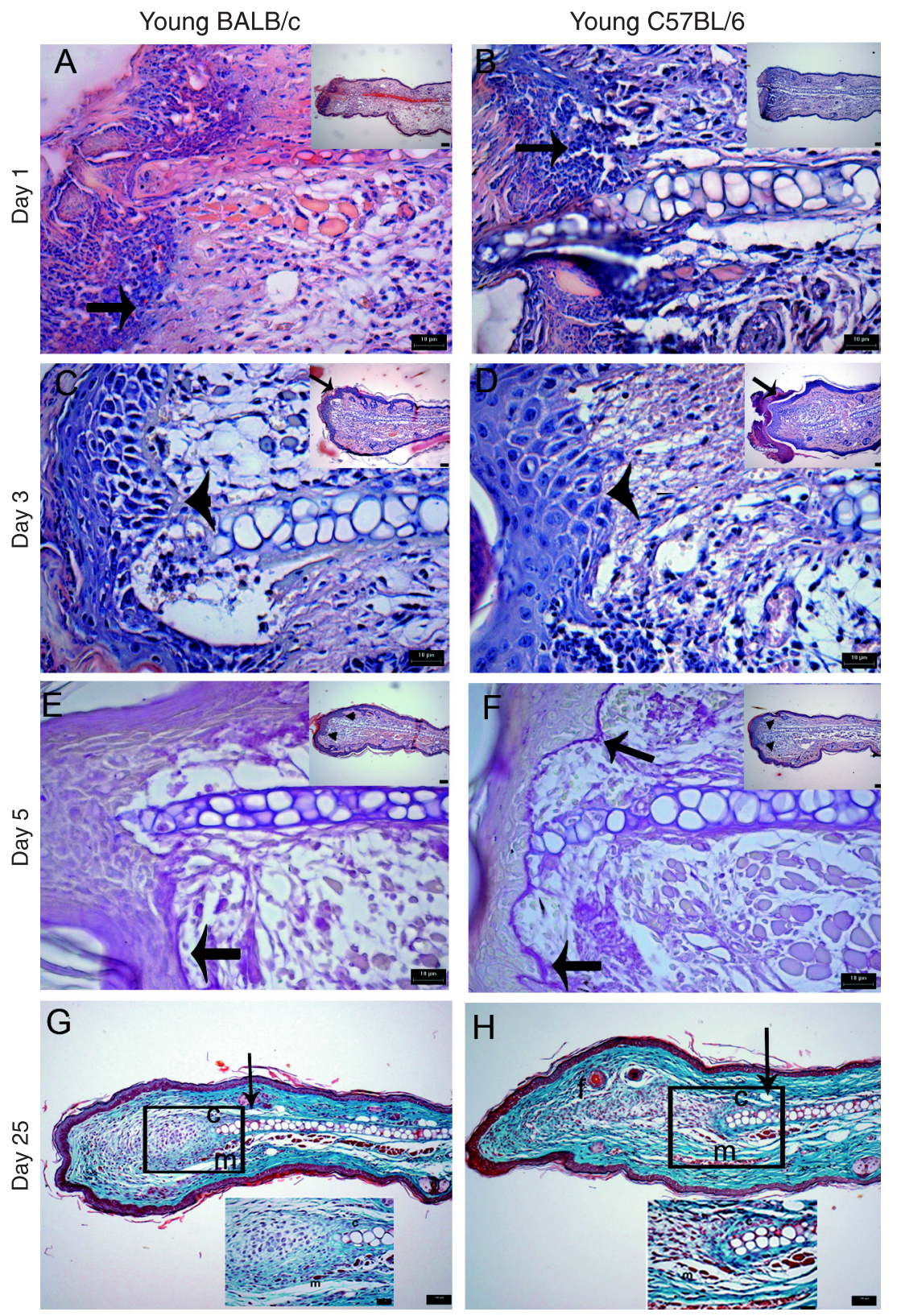

Figure 1. Histological analysis of the wound healing process in the ear of BALB/C and C57BL/6 mice after excisional lesion with a thumb punch. At day 1 ( $A, B$ and insets), the lesion borders show swelling, necrotic tissue and inflammatory infiltrate (arrows). At day 3 (C,D and insets), a fibrin clot is seen (arrows), re-epithelialization has started (arrowheads) and the epithelium is thicker in C57BL/6 mice. Matrix deposition has started in connective tissue of C57BL/6 mice. At day 5, epithelial invagination into connective tissue can be observed in both lineages (inset E,F, arrowheads) but the inflammatory infiltrate is reduced in C57BL/6 mice (inset E,F). The basement membrane is thicker and continuous under the epidermis in C57BL/6 mice compared to that of BALB/c mice (E,F, arrows). At day $25(\mathrm{G}, \mathrm{H})$, cell density in the looser connective tissue is more pronounced in BALB/c mice (inset $G$ ), and the lesion borders are rounded in BALB/C mice $(G)$ while in $C 57 \mathrm{BL} / 6$ mice the lesion borders are cone-shaped and with greater deposition of collagen fibers $(\mathrm{H})$. $G, H$, Arrows indicate cartilage cut edges and squares indicate the regions that were amplified in insets. $c=$ chondrogenesis from pre-existent cartilage; $m=$ myogenesis; $f=$ folliculogenesis. Staining: hematoxylin and eosin (A-D and inset $\mathrm{E}, \mathrm{F})$; periodic acid-Schiff staining and Harris' hematoxylin counterstaining $(E, F)$; Gomori's trichrome $(\mathrm{G}, \mathrm{H})$. Magnification: panels A-F, 400X (bars $=10 \mu \mathrm{m}$ ); panels $\mathrm{G}, \mathrm{H}, 100 \mathrm{X}$ (bars $=100 \mu \mathrm{m}$ ); insets $\mathrm{G}, \mathrm{H}$, 400X (bars $=10 \mu \mathrm{m})$; insets A-F, 100X (bars $=100 \mu \mathrm{m})$. 

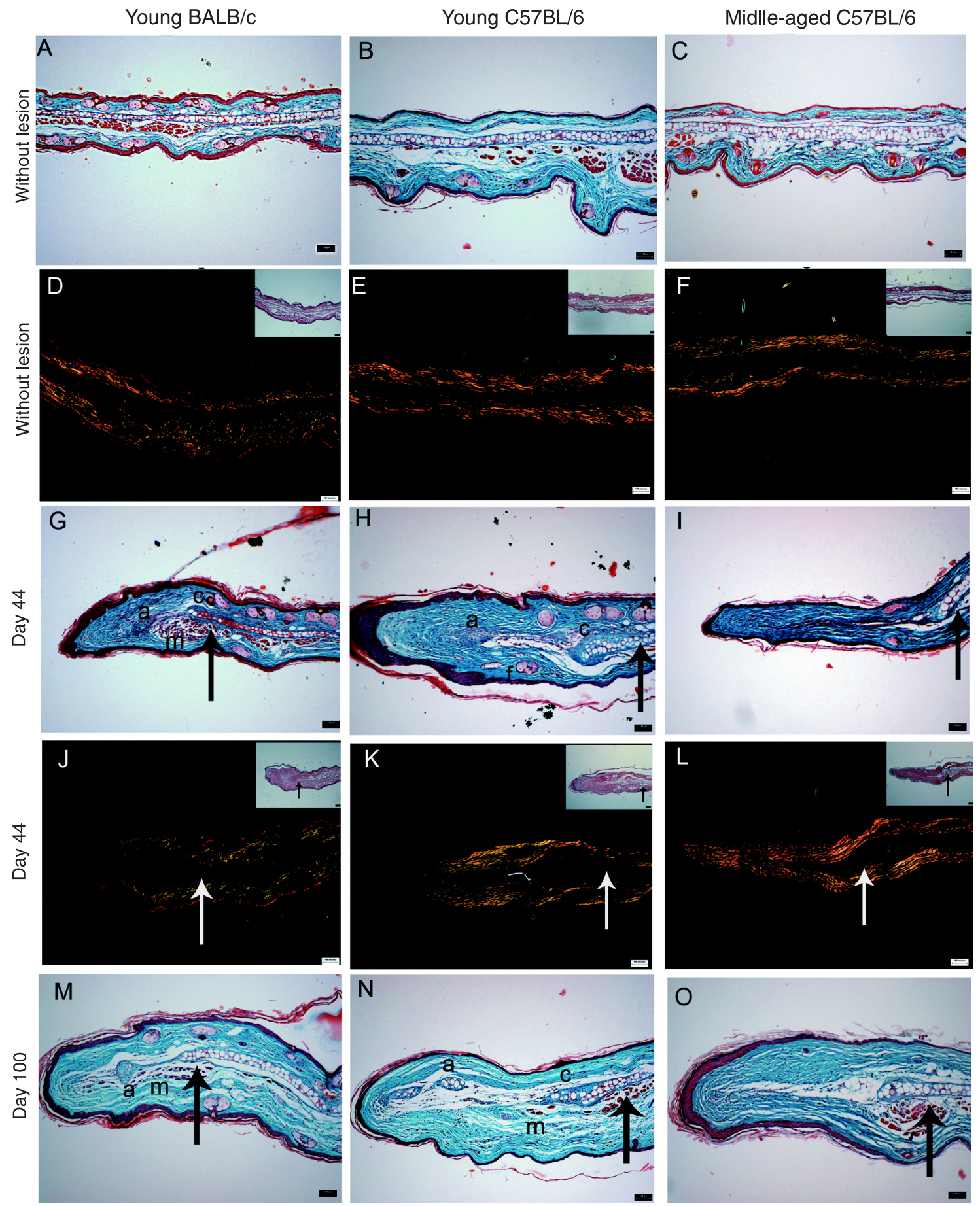

Figure 2. Histological differences in the wound healing process in ears of BALB/c and young and middle-aged C57BL/6 mice 44 and 100 days after excisional lesion with a thumb punch. $A-C$, Central region of ears without lesion. $D-F$, Larger images were obtained by polarized microscopy and insets by polychromatic microscopy of the same sections from normal ear tissues of young BALB/C, young C57BL/6 and middle-aged C57BL/6 mice. At day 44 (G-I) and day 100 (M-O) healing may include cartilage aggregates (a) chondrogenesis (c) from pre-existing cartilage, myogenesis $(\mathrm{m})$, and folliculogenesis (f). $J$-L, Larger images were obtained by polarized microscopy and insets with polychromatic microscopy of the same slides from ear tissues of young BALB/c, young C57BL/6 and middle-aged C57BL/6 mice 44 days after the lesion. Arrows indicate cartilage cut edges. Staining: Gomori's trichrome (A-C, G-I and $\mathrm{M}-\mathrm{O}$ ) and picrosirius red (D-F and J-L). Magnification: pannels A-O and insets, 100X (bars $=100 \mu \mathrm{m}$ ). 
and epithelial down-growths into connective tissue were also observed in both strains. At that time, all the normal layers of the epithelium were already observed in C57BL/6 mice, while in BALB/c mice the epidermis was still thicker. Another prominent difference between C57BL/6 and BALB/C mice that could be observed at day 5 was that the epidermal basement membrane was completely reconstituted in C57BL/6 mice, while it was only starting to be formed in BALB/c mice (Figure 1E,F). Inflammatory infiltrates were still found in connective tissue of both strains with the presence of lymphocytes. However, interestingly, the number of inflammatory cells was reduced in C57BL/6 mice.

By day 25 (Figure $1 \mathrm{G}, \mathrm{H}$ ), the healing ears of both strains showed a regular and thin epithelium devoid of ingrowths. The main differences between strains at day 25 were related to tissue re-organization. The edge of the wound was cone-shaped in C57BL/6 mice (Figure 1H) whereas in $\mathrm{BALB} / \mathrm{c}$ mice the edge of the lesion had a rounded shape with more cells within dermal connective tissue (Figure 1G). Myogenesis occurred in both strains but folliculogenesis was found only in C57BL/6 mice. Chondrogenesis at the cut edge of the cartilage occurred in both strains, but in C57BL/6 mice the extension of recently formed cartilage was greater than in BALB/c mice. All of these aspects of regeneration could still be observed on days 44 and 100 (Figure 2) but a new characteristic found in both strains at day 44 and persisting at day 100 was cartilage formation in aggregates, distant from pre-existing cartilage. From day 44 on, collagen deposition was more pronounced and the number of cells in connective tissue was markedly reduced (Figure 2G,H,M,N).

To evaluate collagen remodeling, ear sections taken 44 days after the wound were stained with picrosirius red and examined by polarized light microscopy to assess the organization of collagen fibrils. It is possible by using the picrosirius-polarization method to distinguish between thick collagen fibers that appear yellow or red and brilliant (strongly birefringent) shining against a dark background, from thin collagen fibers, which display a weak birefringence of a greenish color $(13,14)$. Before the lesion, unmanipulated mice of both strains displayed thick yellow-red fibers under the epithelium and thin green fibers concentrated deeper in the dermis of their ears (Figure 2). Unmanipulated BALB/C mice showed a much less pronounced birefringence and more thin green fibers than C57BL/6 mice (Figure 2D,E). At day 44 after the wound, the healing connective tissue of $\mathrm{BALB} / \mathrm{c}$ mice showed a predominance of thin green fibers deeper in the tissue and some superficial thick fibers (Figure 2J). In contrast, healing connective tissue of young C57BL/6 mice at day 44 presented predominantly thick yellow-red fibers following the direction of cartilage growth while at the tip of the closing edge thin green fibers prevailed (Figure $2 \mathrm{~K})$. These patterns of organization of collagen fibers 44 days after the wound suggest that young BALB/c mice exhibited less remodeling than young C57BL/6 mice, as indicated by thinner, less mature collagen fibers (stained green) that had a greater space between them.

\section{Young and middle-aged C57BL/6 mice}

We also analyzed the process of ear wound healing in middle-aged (40 weeks old) C57BL/6 mice. At day 100, middle-aged C57BL/6 mice displayed a significant increase in percent ear wound closure $(75.31 \pm 23.65 \%)$ in comparison with young C57BL/6 mice (56.47 $\pm 7.99 \%)$, suggesting an improvement in the capacity of healing with age. However, when analyzed histologically (Figure 2), the process of wound healing showed more fibrosis in middleaged C57BL/6 mice than in young mice, with a minimum of cartilage growth and no other signs of skin regeneration such as new hair follicles or sebaceous glands, as observed in young mice of the same strain.

The pattern of collagen deposition in the ears of young and middle-aged C57BL/6 mice was compared by picrosirius red staining under polarized light microscopy (Figure 2E,F and $\mathrm{K}, \mathrm{L}$ ). In middle-aged C57BL/6 mice (Figure 2L), ear wound healing was associated with thick collagen fibers that appeared red and brilliant (strongly birefringent) and densely organized, suggesting a more intense remodeling 44 days after the punch.

\section{Discussion}

In adult mammals, most skin wounds heal by repair and not by regeneration and different types and degrees of scar tissue can be formed depending on the site of wounding (2). The age of the organism also affects the type of healing. Skin wounds in fetal mammals can heal with regeneration and differences in extracellular matrix composition and growth factors or cytokine levels have been associated with the occurrence of regeneration versus repair $(1,7,15)$. The aging process also affects healing as reflected by a temporal delay in wound closure, but whether or not aging affects the quality of healing is not a consensus $(4,12)$. Regardless of many efforts, we still lack a satisfactory answer to why some adult animals regenerate missing body parts and others do not.

We have used here an excisional wound model to study the process of ear wound closure and skin healing in young BALB/c, young C57BL/6 and middle-aged C57BL/6 mice, a model previously used to compare wound closure in MRL mice with other strains considered to be non-regenerative (4-6). The metal thumb punch we used is normally used for lifelong identification of mice; it consists of a blunt metal pin, which is pushed through a metal ring. The wounds were made in the central part of the ear to avoid the "location effect" as described by Williams-Boyce and Daniel Jr. (16) i.e., ear holes in the area proximal to the rabbit head were repaired faster and with more success (78 vs 12\%) than those punched distally. Wound area rather than of wound diameter was measured because the healing lesions are 
not exactly circular.

The wounds in C57BL/6 and BALB/c mice were circumscribed to the area of the metal ring of the punch and no extensive necrosis was observed.

In young mice of both strains, we found re-epithelialization, epithelial down-growth into connective tissue, chondrogenesis, and skeletal muscle formation (Figure 1 ), although the wounds in BALB/c mouse ears closed to a lesser extent. However, regeneration of hair follicles and sebaceous glands occurred in young C57BL/6 mice, but not in BALB/c mice. In summary, histological analysis showed that, in young animals, the healing process was faster in $\mathrm{C} 57 \mathrm{BL} / 6$ than that in BALB/c mice. Collagen remodeling, as well as myogenesis and chondrogenesis, were detected earlier in C57BL/6 mice (Figure 2).

The differences in the extent and quality of ear wound healing between BALB/C and C57BL/6 mice observed in the present study may have been associated with differences in the time of emergence of the new structures. At day 3, the epidermis was thicker in C57BL/6 than in BALB/C mice and at day 5 the basement membrane was already formed in C57BL/6 mice while it was only partially reconstituted in BALB/c mice, in which the process of epithelial stratification was not yet fully developed. Thus, the rapid reorganization of tissues after the wound in C57BL/6 mice may be creating an appropriate place for proliferation and de novo cell differentiation before constraints imposed by adult organization. We did not examine either growth factors or cytokines expressed in healing tissues, nor did we quantify inflammatory cells infiltrated soon after the ear wound, but the inflammatory process was reduced earlier in young C57BL/6 mice than in BALB/c mice. Another important difference between strains may pertain to ear morphology. Unmanipulated BALB/c mice showed a much less pronounced birefringence and a larger amount of thin green fibers than unmanipulated C57BL/6 mice (Figure $2 \mathrm{D}, \mathrm{E}$ ). These two principal variables (altered inflammatory response and ear skin morphology) may influence the outcome of ear wound healing.

In most of the studies reported in the literature, adult BALB/c and C57BL/6 mice do not close their ear wounds to a great extent and signs of regeneration are barely present $(4,17)$. However, using a biopsy punch but not a crude punch, Rajnoch et al. (6) obtained results similar to what we now report for $\mathrm{C} 57 \mathrm{BL} / 6$ mice. On the other hand, in ears perforated with a crude punch they did not find a reduction in wound area, even in MRL mice, which almost closed their ear wounds produced with a clinical biopsy punch (6).

Differences in wound healing observed in adult strains of mice obtained from different sources might derive from genetic drift expected to occur spontaneously in isogenic animal colonies in the course of years. Genetic analyses of capacity for ear tissue regeneration in MRL mice have shown it to be a complex multigenic trait and comparison of the differential expression profiles during the inflammatory stage of repair (24 $\mathrm{h}$ after the lesion) between MRL and C57BL/6J mice suggests that the expression profile in MRL mice exhibited a metabolic shift toward a lower inflammatory response and an enhanced tissue repair beside differences in other genes involved in pathways not previously associated with wound repair (17).

Some reports of tissue regeneration after ear wounds have emphasized epidermal down-growth and the formation of blastema-like structures, consisting of an aggregate of mesenchymal cells near the newly formed epithelium, as being important for the differentiation of new structures $(3-6,18)$. In addition, the re-constitution of the basal membrane was delayed (or absent) in relation to epidermal down-growth (4-6). Similar to what occurs during development when epithelial-mesenchymal interactions result in organogenesis, these interactions would result in newly formed, regenerated structures in adulthood. In our experiments, we identified epidermal down-growth but not a blastema-like structure. We cannot rule out, however, the possibility of a transient and rapid formation of a blastemalike structure at a time that we did not analyze, such as day 4 after the wound. In our results, the absence of a clear blastema-like structure, or its transient appearance, may explain the limited regeneration observed, compared with the complete regeneration observed in rabbits and MRL mice. The presence of the basement membrane while epidermal down-growth is still occurring may also explain the absence of complete regeneration in C57BL/6 mice, as proposed by others (18).

Wound healing in aging organisms involves changes in cell migration and there is a reduction in fibroblasts, inflammatory cells, blood flow, deposition of extracellular matrix, and an increase in its degradation (12). Differences in ear tissue and reduction in stem cells capable to reconstitute the epithelium and skin appendages may also be correlated with impairment of regeneration in aging mice (19).

The higher percentage of wound closure in middle-aged C57BL/6 mice suggested an improvement in healing capacity. However, histological analyses showed that no characteristic of regeneration was found in these animals (Figure $2 \mathrm{I}, \mathrm{O})$; the process of collagen deposition was exacerbated and tissue remodeling was clearly impaired as shown by picrosirius red staining, and they presented a non-organized thicker fiber deposition, with the characteristics of scar tissue (Figure 2L). Other investigators (20) have described a pattern of collagen deposition after excisional wounds in the dorsal skin of old (22 months of age) B6D2F1 mice similar to that observed here in the ear of middle-aged (40 weeks old $=9$ months old) C57BL/6 mice, showing an impairment of the remodeling process.

Attention has been paid to immunological activities in the scar versus the regeneration process during wound healing $(1,7,8)$ and clear immune alterations do occur with aging (11), but no systematic studies of these issues are available. Comparisons of early events in the healing 
process of young and middle-aged mice are very important and should be addressed in additional studies.

To most researchers who work with mice and make ear holes for lifelong identification of individual animals it would sound strange to hear that some mice completely close those holes. But they do. This regenerative capacity

\section{References}

1. Harty M, Neff AW, King MW, Mescher AL. Regeneration or scarring: an immunologic perspective. Dev Dyn 2003; 226: 268-279.

2. Ferguson MW, O'Kane S. Scar-free healing: from embryonic mechanisms to adult therapeutic intervention. Philos Trans $R$ Soc Lond B Biol Sci 2004; 359: 839-850.

3. Goss RJ, Grimes LN. Epidermal downgrowths in regenerating rabbit ear holes. J Morphol 1975; 146: 533-542.

4. Clark LD, Clark RK, Heber-Katz E. A new murine model for mammalian wound repair and regeneration. Clin Immunol Immunopathol 1998; 88: 35-45.

5. Gawronska-Kozak B. Regeneration in the ears of immunodeficient mice: identification and lineage analysis of mesenchymal stem cells. Tissue Eng 2004; 10: 1251-1265.

6. Rajnoch C, Ferguson S, Metcalfe AD, Herrick SE, Willis HS, Ferguson MW. Regeneration of the ear after wounding in different mouse strains is dependent on the severity of wound trauma. Dev Dyn 2003; 226: 388-397.

7. Gurtner GC, Werner S, Barrandon Y, Longaker MT. Wound repair and regeneration. Nature 2008; 453: 314-321.

8. Martin P. Wound healing - aiming for perfect skin regeneration. Science 1997; 276: 75-81.

9. Martin P, D'Souza D, Martin J, Grose R, Cooper L, Maki R, et al. Wound healing in the PU.1 null mouse - tissue repair is not dependent on inflammatory cells. Curr Biol 2003; 13: 1122-1128.

10. Eming SA, Hammerschmidt M, Krieg T, Roers A. Interrelation of immunity and tissue repair or regeneration. Semin is higher in some strains like MRL mice but characteristics of regeneration do occur even in BALB/C and C57BL/6 mice, usually considered to be non-regenerative strains. For those interested in comparing regeneration with what occurs during development, the model of ear wound healing in mice may be interesting.
Cell Dev Biol 2009; 20: 517-527.

11. Linton PJ, Dorshkind K. Age-related changes in lymphocyte development and function. Nat Immunol 2004; 5: 133-139.

12. Gosain A, DiPietro LA. Aging and wound healing. World $J$ Surg 2004; 28: 321-326.

13. Montes GS. Structural biology of the fibres of the collagenous and elastic systems. Cell Biol Int 1996; 20: 15-27.

14. Junqueira LC, Montes GS, Martins JE, Joazeiro PP. Dermal collagen distribution. A histochemical and ultrastructural study. Histochemistry 1983; 79: 397-403.

15. Metcalfe AD, Willis $H$, Beare A, Ferguson MW. Characterizing regeneration in the vertebrate ear. J Anat 2006; 209: 439-446.

16. Williams-Boyce PK, Daniel JC Jr. Regeneration of rabbit ear tissue. J Exp Zool 1980; 212: 243-253.

17. Li X, Mohan S, Gu W, Baylink DJ. Analysis of gene expression in the wound repair/regeneration process. Mamm Genome 2001; 12: 52-59.

18. Williams-Boyce PK, Daniel JC Jr. Comparison of ear tissue regeneration in mammals. J Anat 1986; 149: 55-63.

19. Blanpain C, Lowry WE, Geoghegan A, Polak L, Fuchs E. Self-renewal, multipotency, and the existence of two cell populations within an epithelial stem cell niche. Cell 2004; 118: 635-648.

20. Reed MJ, Karres N, Eyman D, Vernon RB, Edelberg JM. Age-related differences in repair of dermal wounds and myocardial infarcts attenuate during the later stages of healing. In Vivo 2006; 20: 801-806. 